University of Wollongong

Research Online

Faculty of Engineering and Information

Faculty of Engineering and Information

Sciences - Papers: Part A

Sciences

$1-1-1992$

\title{
The response to selection of different procedures for the analysis of early generation variety trials
}

Brian R. Cullis

NSW Department of Agriculture Research Institute, bcullis@uow.edu.au

A. C. Gleeson

NSW Department of Agriculture Research Centre

F. M. Thomson

NSW Department of Agriculture Research Institute

Follow this and additional works at: https://ro.uow.edu.au/eispapers

Part of the Engineering Commons, and the Science and Technology Studies Commons

Research Online is the open access institutional repository for the University of Wollongong. For further information contact the UOW Library: research-pubs@uow.edu.au 


\title{
The response to selection of different procedures for the analysis of early generation variety trials
}

\begin{abstract}
A simulation study was conducted to determine the relative response to selection of a one-dimensional spatial analysis of field experiments (SAFE) specifically adapted for early generation trials. The primary purpose of the analysis in these trials is to obtain accurate estimates of genotypic effects of the unreplicated test lines by adjusting for local variation, using either the yields of all neighbouring plots or the yields of neighbouring plots of (replicated) check varieties. The response to selection of the SAFE analysis, relative to the use of unadjusted yields of test line plots, was consistently greater than the relative response to selection of check-plot-only analyses. The relative response to selection of SAFE increased as the percentage of trend variance increased.
\end{abstract}

\section{Keywords}

variety, trials, early, generation, analysis, different, procedures, response, selection

Disciplines

Engineering | Science and Technology Studies

\section{Publication Details}

Cullis, B. R., Gleeson, A. C. \& Thomson, F. M. (1992). The response to selection of different procedures for the analysis of early generation variety trials. The Journal of Agricultural Science, 118 (2), 141-148. 


\title{
The response to selection of different procedures for the analysis of early generation variety trials
}

\author{
B. R. CULLIS', A. C. GLEESON ${ }^{2}$ AND F. M. THOMSON ${ }^{1}$ \\ ${ }^{1}$ NSW Department of Agriculture Research Institute, Wagga Wagga, NSW 2650, Australia \\ ' NSW Department of Agriculture Research Centre, Tamworth, NSW 2340, Australia
}

(Revised MS received II September 199I)

\begin{abstract}
SUMMARY
A simulation study was conducted to determine the relative response to selection of a onedimensional spatial analysis of field experiments (SAFE) specifically adapted for early generation trials. The primary purpose of the analysis in these trials is to obtain accurate estimates of genotypic effects of the unreplicated test lines by adjusting for local variation, using either the yields of all neighbouring plots or the yields of neighbouring plots of (replicated) check varieties. The response to selection of the SAFE analysis, relative to the use of unadjusted yields of test line plots, was consistently greater than the relative response to selection of check-plot-only analyses. The relative response to selection of SAFE increased as the percentage of trend variance increased.
\end{abstract}

\section{INTRODUCTION}

Because of the large number of test lines involved in the early stages of a plant-breeding programme and the selection of relatively few of these test lines for further testing, reliable selection of those test lines with the highest genetic potential is very important. At this stage of the programme, seed supply is limited and because of the magnitude of the genotype $x$ environment interaction it is current practice in Australia to sow only one plot of each test line at each site (although two replicates of each test line are occasionally included). The test plots are generally interspersed with check plots, sown to standard varieties and regularly spaced along each of several rows. Plant breeders at NSW Agriculture typically conduct trials with $>500$ test lines, requiring $c .10$ blocks (rows) of c. 50 plots (columns). In such large ficld experiments, it is important that an effective method of local yield control be applied to the yields of test plots to maintain reasonable genetic progress.

For replicated field trials, there has been considerable statistical research into the efficiency of different designs and methods of analysis. Patterson \& Hunter (1983) showed that the use of incomplete block designs or alpha designs for the design and analysis of replicated variety trials resulted in an average reduction of $30 \%$ in the variance of varietal yield differences relative to randomized complete block analysis. In a study of 1019 replicated cereal variety trials grown over 3 years in four states of Australia, Cullis \& Gleeson (1989) showed that the use of spatial analysis of field experiments (Gleeson \& Cullis 1987) resulted in an average reduction of $42 \%$ in the variances of varietal yield differences compared to randomized complete block analysis. Of the 1019 trials, 239 were incomplete block designs and the average reduction in the variances of varietal yield differences for incomplete block analysis (with recovery of interblock information) was $33 \%$; the average reduction for spatial analysis of these 239 trials was $44 \%$.

For the analysis of unreplicated early generation variety trials there has been very little progress. A survey of Australian plant breeders in 1988 showed that the most frequently used analyses were the method of moving means (Richey 1926) and an adjustment proposed by Yates (1940) using a weighted average of check plots. Both procedures subtract a local fertility index from each test plot yield. For the moving means procedure, the fertility index is calculated as the mean of the $k / 2$ row adjacent plots whereas Yates's method uses a weighted average of the row adjacent check plots.

Lin \& Poushinsky (1985) proposed a modified augmented design (type 2) for unreplicated early generation variety trials. The design is constructed in a split-plot arrangement with main plots laid out in several rows. Each main plot consists of a central check plot on each side of which there are several subplots to which test lines are allocated at random. Additional check varieties (including the same variety used for the central plot if desired) may be allocated at random to a subplot for an arbitrary number of 
main plots. These extra check plots are used to estimate subplot error in the subsequent analysis.

Kempton (1984) summarized previous comparisons of check-plot-only methods such as that of Yates (1940) with the method of moving means. He found that, generally, results favoured the use of moving means. Kempton pointed out, however, that because different variants of the methods were used in different studies, and the comparison of methods was based on error mean squares which is not strictly valid, interpretation of these results is not straightforward. Kempton used a model describing correlations between plots to compare check-plot-only and moving means methods. This demonstrated that the method of moving means achieves a consistently higher expected genetic gain than check-plot-only methods, irrespective of check plot frequency and genetic variance. Despite this result, he recommended the use of check plots at a frequency not greater than 1:5, as the inclusion of these plots can be of practical use and does obviate the need for randomization of test lines.

Cullis et al. (1989) extended the one-dimensional spatial analysis for field experiments to analyse unreplicated early generation variety trials. In their method, the variance parameters relating to genotype, trend and error are estimated using residual maximum likelihood (REML). Best linear unbiased predictors (BLUPs) of the test line genotypic effects are then calculated. The BLUPs of the test line genotype effects are obtained by subtracting from the test line plot yield a weighted average of neighbouring plot yields, the weights being functions of the estimated variance parameters. Thus the SAFE method of adjustment can be regarded as a weighted moving mean adjustment in which the weights are determined according to the relative magnitude of the genetic, trend and error variances. Cullis et al. (1989) showed that the method had reasonably low levels of bias for estimating variance parameters (i.e. genetic and error) in the presence of field trends, with the added advantage of being able to estimate fixed effects such as covariates both efficiently and simultaneously.

This paper presents results of a comparison, based on the relative response to selection, of the SAFE procedure, Yates's (1940) procedure and the analysis of variance procedure proposed by Lin \& Poushinsky (1985). Results from the method of moving means are also included for an approximate comparison with the check-plot-only methods and SAFE. The comparison is based on simulated data using trends from 20 sets of uniformity data.

\section{MATERIALS AND METHODS}

\section{Uniformity data}

Twenty sets of uniformity data were used to produce trends for the basis of the simulation study. These data sets are described in Table I and cover a range of field crops with plot sizes which are representative of the plot sizes used in unreplicated early generation variety trials. Each set of uniformity data was smoothed using the five-point moving median method, known as 4(3RSR)2H (Tukey 1977) and available in the computer package S (Becker et al. 1988). The smoothing was performed within rows of plots aligned with their longest side in common. This smoothing partitioned the total variance into two components, trend variance $\left(\sigma_{r}^{2}\right)$ and error variance $\left(\sigma_{e}^{2}\right)$. Table 1 presents a summary of the percentage trend variance relative to the total variance, $R_{T}^{2}=100 \times \sigma_{T}^{2} /\left(\sigma_{T}^{2}+\sigma_{e}^{2}\right)$. For the 20 sets of data, $R_{T}^{2}$ varied from 33 to $89 \%$ with an average value of $65 \%$. Figure 1 presents a plot of the fourth set of uniformity data and the smoothed trend derived from it.

\section{Statistical methods}

Data were generated by taking the value of the trend for each plot, adding a random error and then adding either a test line effect or a check variety effect. A modified augmented design (type 2) was used as the basis for the allocation of check plots and test plots. To simplify the analyses, especially for the moving means and Yates's methods, only one check plot variety was used, although in practice we would advocate the use of two or more check varieties. Check plots were assigned at two check plot frequencies $(F=1: 5$ and $1: 9)$ and for each frequency three levels of genetic/error variance ratio $\left(\lambda=\sigma_{g}^{2} / \sigma_{e}^{2}\right.$ $=0.5,1.0,5.0$ ) were chosen. For a frequency of $1: 5$ there are four test plots per main plot whilst for a frequency of 1:9 there are eight test plots per main plot. Trend maps were trimmed to the appropriate size, when necessary, by deletion of the plots from one end of each row. Random test line genotypic effects were generated as independent and identically distributed normal variates with mean zero and variance $\sigma_{g}^{2}$. Random errors were generated as independent and identically distributed normal variates with mean zero and variance $\sigma_{e}^{2}$. Test line effects and errors were generated independently. Values of $\sigma_{e}^{2}$ were set at the values calculated for each set of uniformity data. The check variety was assumed to have a mean value of $80 \%$ of the average of the test lines. Fifty repetitions were generated and analysed for each of the two check plot frequencies, three genetic variance ratios and 20 sets of uniformity data, representing a total of 6000 simulated data sets.

Each simulated data set was subjected to five methods of analysis. These were as follows:

(1) SAFE - the one-dimensional spatial analysis developed by Cullis et al. (1989). This method assumes the trend in each row is represented approximately by a first-order integrated process, termed an ARIMA $(0,1,0)$ (Box \& Jenkins 1970). 
Response to selection in early generation variety trials

Table 1. Description of sets of uniformity data

\begin{tabular}{cllcccc}
\hline Dataset & Source & Crop & Plot size & Rows & Columns & $R_{T}^{2}$ \\
\hline 1 & NSWAF* & Barley & $1 \cdot 6 \times 9 \cdot 5$ & 26 & 9 & 83 \\
2 & NSWAF & Barley & $1 \cdot 6 \times 7$ & 20 & 12 & 85 \\
3 & NSWAF & Wheat & $1 \cdot 8 \times 9$ & 20 & 11 & 52 \\
4 & NSWAF & Wheat & $1 \cdot 8 \times 9$ & 30 & 6 & 75 \\
5 & NSWAF & Wheat & $2 \times 20$ & 24 & 8 & 84 \\
6 & WADA & Wheat & $1 \cdot 8 \times 10$ & 20 & 8 & 43 \\
7 & WADA & Wheat & $1 \cdot 8 \times 10$ & 20 & 8 & 68 \\
8 & WADA & Wheat & $1 \cdot 8 \times 10$ & 20 & 8 & 59 \\
9 & WADA & Wheat & $1 \cdot 8 \times 10$ & 40 & 4 & 50 \\
10 & WADA & Wheat & $1 \cdot 8 \times 10$ & 20 & 8 & 62 \\
11 & WADA & Wheat & $1 \cdot 8 \times 10$ & 40 & 4 & 33 \\
12 & WADA & Wheat & $1 \cdot 8 \times 10$ & 40 & 5 & 84 \\
13 & WADA & Wheat & $1 \cdot 8 \times 5$ & 46 & 6 & 74 \\
14 & WADA & Wheat & $1 \cdot 8 \times 10$ & 19 & 8 & 49 \\
15 & WADA & What & $1 \cdot 8 \times 10$ & 20 & 8 & 84 \\
16 & WADA & Wheat & $1.8 \times 5$ & 46 & 6 & 50 \\
17 & BSES $\$$ & Sugar & $1.45 \times 10$ & 22 & 17 & 52 \\
18 & Williams \& Luckett & Cotton & $1 \times 12$ & 24 & 12 & 65 \\
19 & Kempton \& Howes & Wheat & $1.5 \times 4.5$ & 28 & 7 & 89 \\
20 & Williams \& Luckett & Barley & $0.75 \times 3 \cdot 3$ & 48 & 15 & 67 \\
\hline \hline
\end{tabular}

* New South Wales Agriculture \& Fisheries.

$\dagger$ Western Australian Department of Agriculture.

$\ddagger$ Bureau of Sugar Experiment Stations.

$R_{r}^{2}$, denotes percentage trend variance, see text.
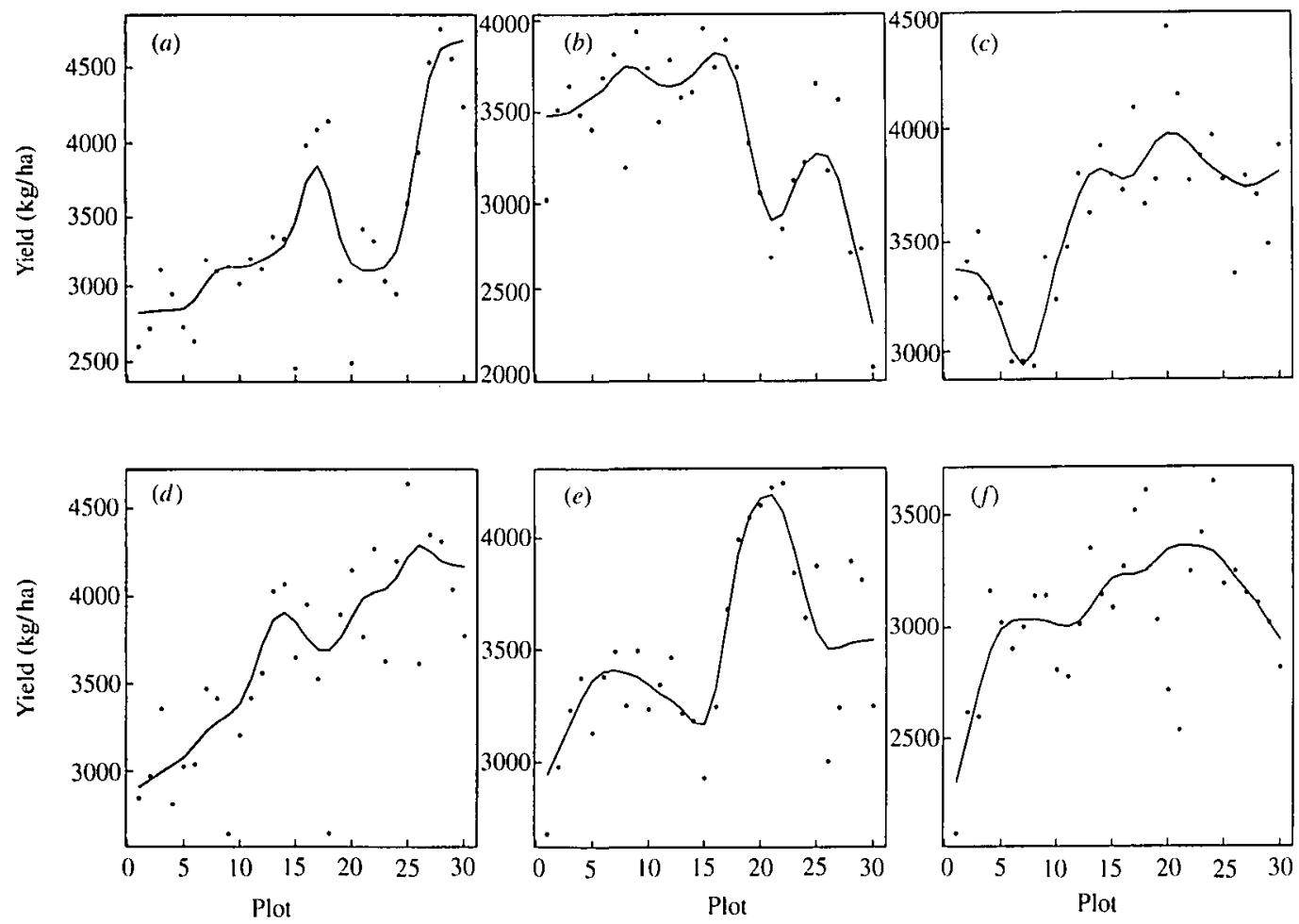

Fig. 1. Yield and smoothed trend for data set 4 of Table 1; (a) Row 1, (b) Row 2, (c) Row 3, (d) Row 4, (e) Row 5 and (f) Row 6. 
(2) YWA - the method proposed by Yates (1940) and widely adopted by plant breeders. This method subtracts from the yield of each test plot a fertility index calculated as the (distance) weighted average of row adjacent check plots.

(3) LP - the analysis of variance method proposed by Lin \& Poushinsky (1985). For this method, row and column effects are first calculated from a row + column analysis of the central plots in each main plot. Each test line within a main plot then has the row and column effects for that main plot subtracted from its yield. Kempton (1984) has shown that in order to maintain genetic gain, the frequency of the check plots should be kept below 1 in 5 . Consequently the option suggested by Lin \& Poushinsky (1985) of including additional check plots in each main plot was not adopted in this study, hence their Method 3 was not applicable. Eighteen of the 20 data sets used in this study were also used by Cullis \& Gleeson (1991), who showed that for 17 of these 18 , the lowest error mean square (as a percentage of the total mean square) was achieved with a row + column analysis. Thus for these data sets, the LP method used in this study is likely to be the most efficient of the three methods proposed by Lin \& Poushinsky (1985).

(4) \& (5) MM5, MM7 - the method of five- or sevenpoint moving means first proposed by Richey (1926). This method is also widely used by plant breeders for the analysis of unreplicated early generation variety trials and subtracts from the yield of each test plot a fertility index calculated as the mean of the test plot plus two or three row adjacent plots on either side of the relevant test plot. For plots at the end of the row, the moving mean is calculated as though 'plots' beyond the end of the row are reflections about the end, of the internal plots.

For Methods 2, 3, 4 and 5, the estimated mean yield difference between the test plots and the check plots was subtracted from each check plot before adjustment.

Kempton (1984) suggested that an appropriate measure to compare the effectiveness of different methods of analysis of unreplicated early generation variety trials, for various check plot frequencies, is the Expected Genetic Gain. This accounts both for the accuracy of the method in estimating the true test line effects and for the cost of including extra check plots at the expense of test plots, assuming that the total number of plots is fixed. The Expected Genetic Gain is given by $\Delta=i h^{2}$, where $i$ is the selection intensity and $h^{2}$ is the heritability. SAFE is the only method that estimates variance components (and therefore heritability) directly. Falconer (1981) suggested the Expected Response to Selection $(R)$,

$$
R=i h \sigma_{\vartheta}=i \sigma_{g} \operatorname{corr}(u, \hat{u})
$$

where $i$ and $h$ are as previously defined, $\operatorname{corr}(u, \hat{u})$ denotes correlation between the true genetic effect $u$ and its estimate $\hat{u}$ and $\sigma_{g}$ is the square root of the additive genetic variance $\sigma_{g}^{2}$, as a measure to compare different methods of selection. We therefore define the (observed) Relative Response to Selection (RRS) by

$$
R R S=i_{m} \operatorname{corr}(u, \hat{u}) /\left(i_{r} \operatorname{corr}(u, y)\right)
$$

where $u$ is the vector of known genotypic effects, $\hat{u}$ is the vector of adjusted test plot yields for method $m$ and $y$ is the vector of raw test plot yields. The selection intensity $i_{m}$ differs from $i_{r}$ for Methods 1,2 and 3 but $i_{m}=i_{r}$ for Methods 4 and 5. This is consistent with the approach of Kempton (1984), in which it is assumed that the trial size is fixed and it is also assumed that the number of selected lines is $10 \%$ of the total number of plots when there are no check plots. Therefore, for Methods 1, 2 and 3, $i_{m}=1.647$ and 1.699 for the two check plot frequencies of 1:5 and $1: 9$ respectively, and $i_{m}=i_{r}=1.755$ for Methods 4 and 5, regardless of check plot frequency. $R R S$ is therefore a conservative estimate of the Relative Response to Selection for Methods 1, 2 and 3 and favours the method of moving means as the same design (including check plots) was used for all the analyses.

\section{RESULTS AND DISCUSSION}

\section{Variance component estimation of SAFE}

Table 2 lists the percentage bias ((estimatedtrue)/true $\times 100$ ) of the REML estimates of genetic $\left(\sigma_{g}^{2}\right)$ and error $\left(\sigma_{e}^{2}\right)$ variance for each of the six combinations of two check plot frequencies $(F)$ and three levels of genetic/error variance ratio $\left(\lambda=\sigma_{g}^{2} / \sigma_{e}^{2}\right)$. The values for each combination are the mean values of the 50 repetitions for each of the 20 sets of uniformity data. Cullis et al. (1989) described the iterative estimation procedure used to obtain SAFE estimates of variance parameters. Convergence was achieved to valid parameter values for $c .95 \%$ of the repetitions. For most of the remaining repetitions, the SAFE estimate of one of the variance parameters $(\lambda)$ occurred at the boundary of the parameter space (i.e. $\lambda=0$ ). This usually occurred at the lowest value for $\lambda$, where the non-negativity constraint on $\lambda$ appears to have induced a positive bias for $\hat{\sigma}_{v}^{2}$ (Table 2). For

Table 2. Variance component estimation using SAFE,

\begin{tabular}{|c|c|c|c|}
\hline & $\lambda=0.5$ & $\lambda=1.0$ & $\lambda=5 \cdot 0$ \\
\hline \multicolumn{4}{|c|}{ Genetic variance $\left(\sigma_{o}^{2}\right)$} \\
\hline$F=1: 5$ & 17 & 3 & 1 \\
\hline$F=1: 9$ & 20 & 8 & 3 \\
\hline \multicolumn{4}{|c|}{ Error variance $\left(\sigma_{e}^{2}\right)$} \\
\hline$F=1: 5$ & -19 & -15 & -7 \\
\hline$F=1: 9$ & -23 & -17 & -7 \\
\hline
\end{tabular}
mean percent bias for 1000 repetitions 

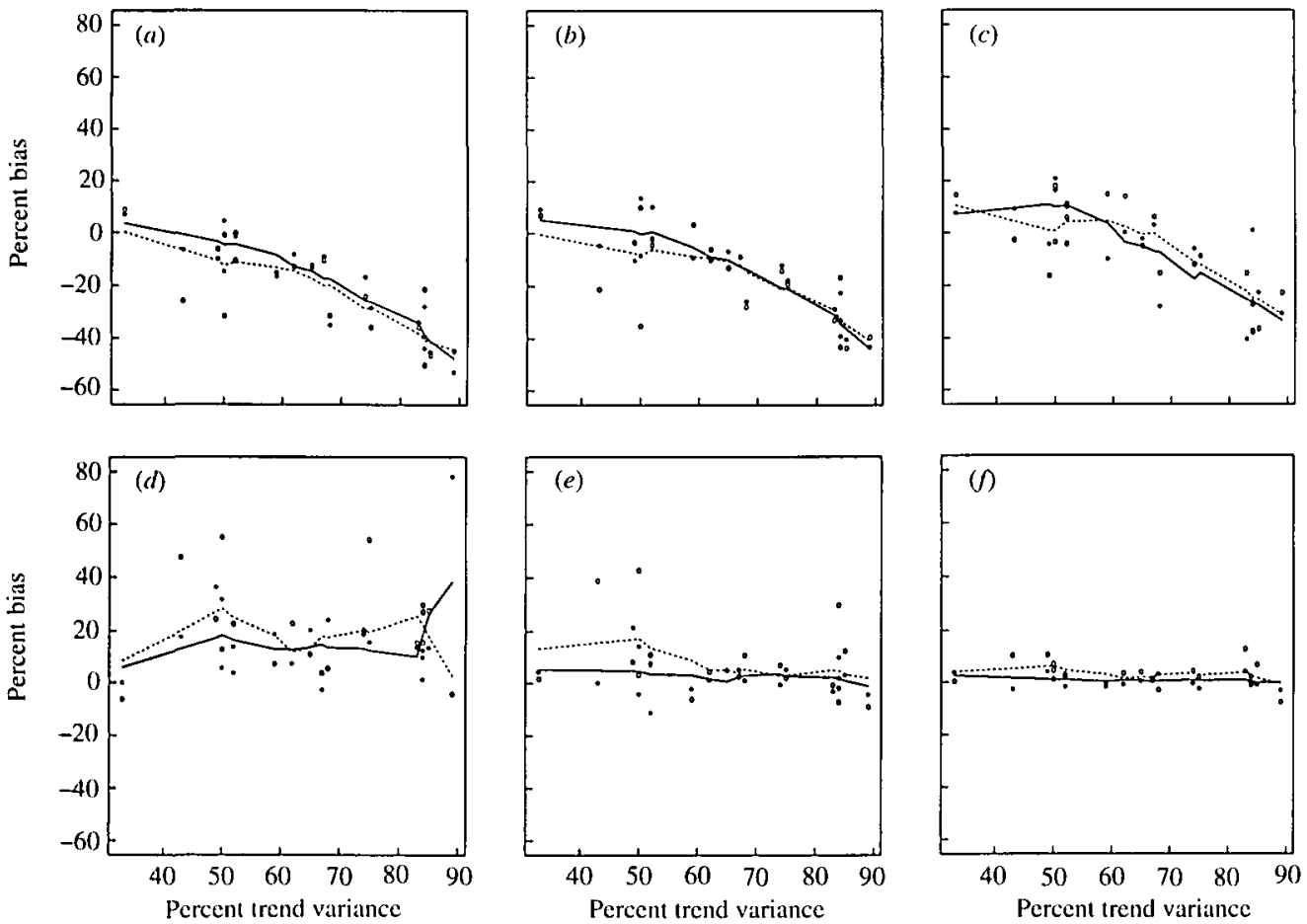

Fig. 2. Percentage bias of REML estimators of error and genetic variance for two check plot frequencies $(F=1: 9$, $\cdots, O)$ and $(F=1: 5,-, O)(a)$ error variance, $\lambda=0.5(b)$ error variance, $\lambda=1.0(c)$ error variance, $\lambda=5.0(d)$ genetic variance, $\lambda=5.0(e)$ genetic variance, $\lambda=1.0$ and $(f)$ genetic variance, $\lambda=5.0$.

Table 3. Relative Response to Selection of five methods of adjustment (see text)

\begin{tabular}{|c|c|c|c|c|c|c|c|}
\hline$\lambda$ & $F$ & $\operatorname{corr}(u, y)$ & SAFE & YWA & LP & MM5 & MM7 \\
\hline \multirow[t]{2}{*}{0.5} & $1: 5$ & 0.371 & 1.41 & $1 \cdot 26$ & $1 \cdot 12$ & 1.48 & 1.48 \\
\hline & $1: 9$ & 0.375 & 1.41 & $1 \cdot 13$ & 1.09 & 1.43 & 1.43 \\
\hline \multirow{2}{*}{1.0} & $1: 5$ & 0.490 & 1.29 & 1.18 & 1.08 & 1.35 & 1.36 \\
\hline & $1: 9$ & 0.495 & 1.31 & $1 \cdot 10$ & 1.07 & 1.32 & 1.33 \\
\hline \multirow[t]{2}{*}{5.0} & $1: 5$ & 0.773 & 1.07 & 1.05 & 1.01 & 1.08 & $1 \cdot 10$ \\
\hline & $1: 9$ & 0.775 & 1.08 & 1.03 & 1.02 & 1.06 & 1.09 \\
\hline
\end{tabular}

$\lambda=0.5$, the median bias was $14 \%$ for $F=1: 5$ and $18 \%$ for $F=1: 9$. The bias of $\hat{\sigma}_{g}^{2}$ appears only marginally larger for $F=1: 9$ than for $F=1: 5$. Figure 2 presents a graph of the percentage bias of $\hat{\sigma}_{g}^{2}$ plotted against the percentage trend variance $\left(R_{T}^{2}\right)$ for the 20 uniformity sets; results are presented for each check plot frequency $\left(1: 5\right.$ and 1:9). Estimates of $\hat{\sigma}_{g}^{2}$ are remarkably stable over the range of percentage trend variance in these data sets.

The REML estimator of $\sigma_{e}^{2}$ was negatively biased in all cases (Table 2). The greatest bias occurred for $F=$ $1: 9$ and $\lambda=0.5$. A simulation study conducted by Cullis et al. (1989) showed no consistent negative bias for $\hat{\sigma}_{e}^{2}$ when the trend of the simulated data was generated as an ARIMA $(0,1,0)$ rather than, as in this study, obtained from smoothing uniformity data. This discrepancy between the studies raises the question of the adequacy of ARIMA $(0,1,0)$ processes to describe trends in these types of field trials, and reinforces the need for diagnostics to determine an appropriate trend model for each data set. Cullis et al. (1991) have developed diagnostics to determine the adequacy of the proposed trend model, but their results are only applicable to replicated field experiments. They are, however, currently being extended for the identification of trend models in unreplicated early generation variety trials. The graph in Fig. 2 of the bias in $\hat{\sigma}_{e}^{2}$ plotted against the percentage trend 
Table 4. Number (out of 20) of ineffective adjustments, i.e. when $R R S \leqslant 1$

\begin{tabular}{rcccccc}
\hline$\lambda$ & $F$ & SAFE & YWA & LP & MM5 & MM7 \\
\hline 0.5 & $1: 5$ & 0 & 6 & 3 & 0 & 0 \\
& $1: 9$ & 0 & 8 & 6 & 0 & 0 \\
1.0 & $1: 5$ & 1 & 8 & 6 & 0 & 0 \\
& $1: 9$ & 0 & 9 & 8 & 0 & 0 \\
5.0 & $1: 5$ & 8 & 10 & 12 & 8 & 6 \\
& $1: 9$ & 6 & 10 & 11 & 10 & 8 \\
\hline \hline
\end{tabular}

variance illustrates that the negative bias of $\hat{\sigma}_{e}^{2}$ increases with increasing within-site variability. There appears to be little effect of check plot frequency on this bias.

\section{Accuracy of the genotypic effect prediction}

Table 3 presents a summary of the mean of Relative Response to Selection for each of the five methods of adjustment. An analysis of variance was performed in which the 20 sets of uniformity data were considered as 'blocks' and the six factorial 'treatments' were two levels of $F \times$ three levels of $\lambda$. These analyses showed significant differences in Relative Response to Selection between levels of $\lambda$ for all five methods of adjustment. There were also significant differences between the two check plot frequencies, for YWA and LP, and an interaction between $\lambda$ and $F$ for YWA.

The Relative Response to Selection of the method of moving means was slightly higher than that for the SAFE method when $\lambda=0.5$ or 1.0 and $F=1: 5$; however, there was little difference between these methods for $\lambda=5.0$ for either check-plot frequency. This supports the results of Kempton (1984), that the optimum number of plots for the calculation of the moving mean depends on the relative size of the genetic variance. SAFE (and MM5 and MM7) consistently outperformed the check-plot-only adjustment methods YWA and LP.

The Relative Response to Selection of YWA was significantly reduced by reducing the check plot frequency, with the largest reduction occurring for $\lambda=0.5$. The Relative Response to Selection of LP was less than that of YWA for all levels of $\lambda$ and $F$.

The arithmetic mean Relative Response to Selection of SAFE over all levels of genetic/error variance ratios and both check plot frequencies was 1.260, compared with a mean Relative Response to Selection of 1.125 and 1.065 for YWA and LP respectively.
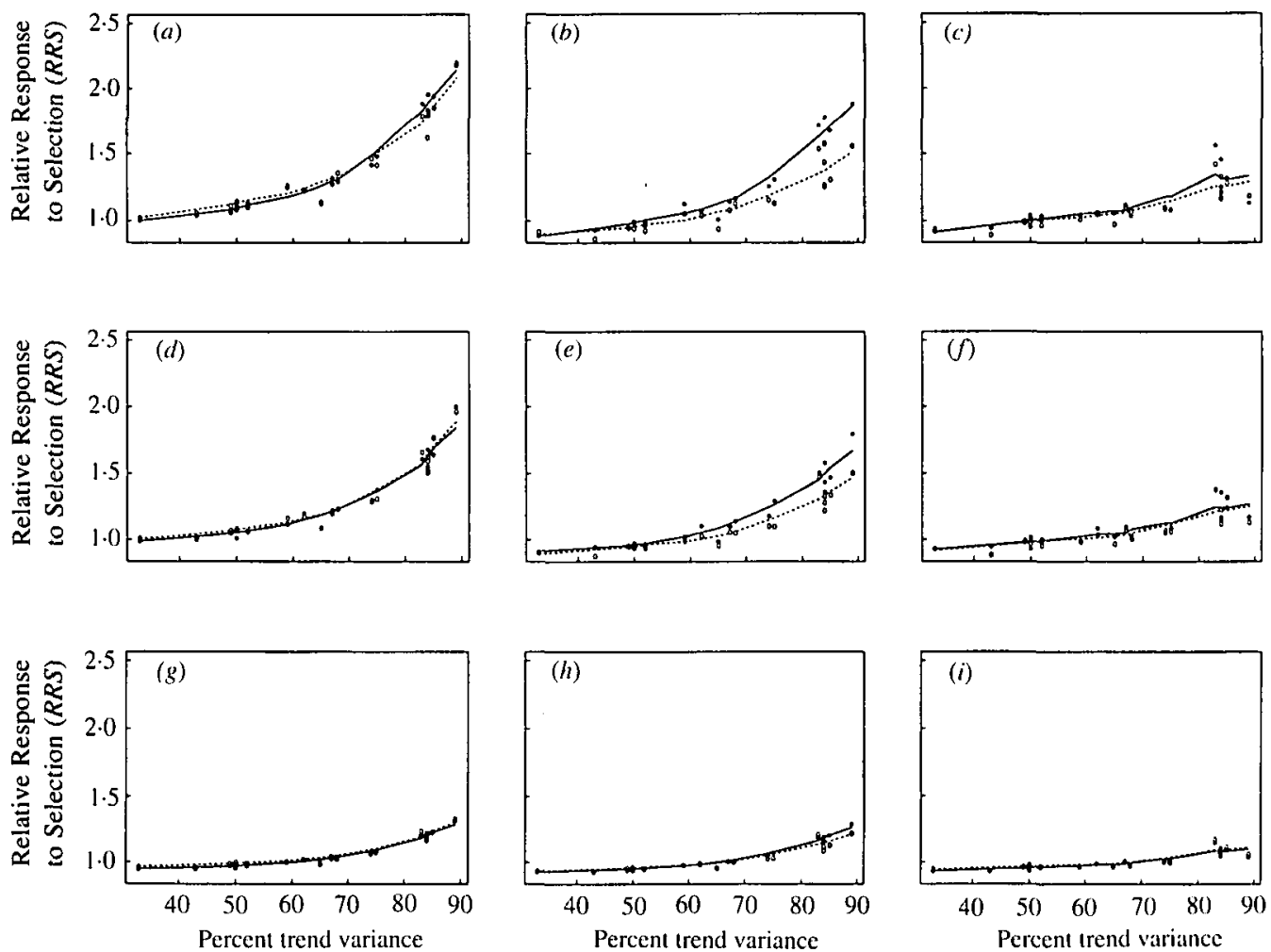

Fig. 3. Relative Response to Selection $(R R S)$ for three methods of adjustment and two check plot frequencies $(F=1: 9$, $\cdots, O)$ and $(F=1: 5,-, 0)(a)$ SAFE, $\lambda=0.5(b)$ YWA, $\lambda=0.5(c)$ LP, $\lambda=0.5(d)$ SAFE, $\lambda=1.0(e)$ YWA, $\lambda=1 \cdot 0$ (f) LP, $\lambda=1.0(\mathrm{~g}) \mathrm{SAFE}, \lambda=5.0(h) \mathrm{YWA}, \lambda=5.0$ and (i) LP, $\lambda=5.0$. 
Table 4 presents a summary of the number of data sets (out of 20) for which the Relative Response to Selection was $\leqslant 1$, that is, those for which the method of adjustment was ineffective. This indicates that SAFE was ineffective on only one occasion for $\lambda=0.5$ and 1.0 , compared to a total of 31 and 23 occasions in which YWA and LP were ineffective for the same values of $\lambda$. All methods were ineffective on more occasions for $\lambda=5 \cdot 0$, the most effective methods being SAFE and MM5.

Figure 3 illustrates the Relative Response to Selection of three methods (SAFE, YWA and LP) at two check plot frequencies, as the percentage trend variance increases. The Relative Response to Selection of SAFE increases markedly as percentage trend variance increases. The Relative Response to Selection of YWA and LP similarly increases with increasing percentage trend variance but at a much slower rate than for SAFE. Figure 3 highlights the major difference between SAFE (and moving means) and the check-plot-only adjustment methods YWA and LP; the lower effectiveness for YWA and LP when there is substantial trend variance.

The Relative Response to Selection of all methods was greatest for lower genetic/error variance ratios. These lower ratios are more common in unreplicated early generation variety trials conducted by plant breeders in Australia and so it is likely that in their trials the Relative Response to Selection of SAFE will be $>1.260$ in most cases.

The Relative Response to Selection of SAFE was not seriously affected by lowering the check plot frequency from 1:5 to $1: 9$, whereas the Relative Response to Selection of YWA, and to a lesser extent LP, was reduced for a check plot frequency of 1:9. From this result, when using SAFE it appears reasonable to recommend a check plot frequency of less than $I$ in 5.

The high proportion of data sets for which YWA and LP were ineffective (i.e. reduced the response to sclection, irrespective of check plot frequency) is a major concern. This result indicates that these checkplot-only methods can reduce response to selection even for a check plot frequency of 1 in 5, depending on the percentage trend variance. Kempton (1984) recommended a maximum check plot frequency of 1 in 5. This frequency, however, reduced the response to selection for $40 \%$ of the data sets when using the YWA method of adjustment. His results were based on two uniformity data sets, one of which is included in this study (19) and, not surprisingly, has the largest percentage trend variance.
It is clear from Fig. 3 that the benefits of SAFE (and moving means) adjustment are greater for trials with a high percentage trend variance. Alternatively, if error variance can be reduced through improved experimental procedures, the benefits of SAFE (and moving means) will be increased further. This is not the case for YWA and LP, however, where increases in Relative Response to Selection are not as large for data sets with more trend variance.

Comparisons of the five methods in this paper are based on the same design (modified augmented (type 2)). Clearly, for moving means this is not strictly valid, as check plots are treated as test line plots, with no adjustment to the selection intensity. This is illustrated by the consistently lower $R R S$ of the moving means procedures for the 1:9 check plot frequency. Although the results in Table 3 indicate that moving means generally outperforms SAFE, the ratios of the $R R S$ between SAFE and the moving means methods were less than the ratios of the selection intensities.

The similar performance of SAFE and the moving means methods prompts us to re-emphasise the importance of randomization in the design of unreplicated early generation variety trials. For the SAFE analysis, a genetic relationship matrix $A$ (Cullis et al. 1989) can be specified for the test lines. Plant breeders in Australia rarely select within families or have a sound knowledge of the genetic relationships to include in $A$, so it is usual to assume that the test lines are independent. Test lines should therefore be allocated to test plots randomly.

The low levels of bias of the SAFE estimation of $\sigma_{a}^{2}$, particularly when $\lambda>0.5$, are encouraging, despite the negative bias of the estimator of $\sigma_{e}^{2}$. Most interest in unreplicated early generation variety trials is focused on the predicted test line genotypic effects for which this study has shown SAFE to outperform the check-plot-only methods of adjustment, despite the questionable adequacy of ARIMA $(0,1,0)$ processes for describing the trend in highly variable sites. This is in accordance with the general observation that improved estimates of treatment effects can be obtained using only rough approximations to the error structure (Martin 1985). It is expected, however, that when suitable diagnostics are developed for the identification of the most appropriate ARIMA trend model, similar to those obtained by Cullis et al. (1991), for replicated field experiments, the bias in error variance estimation may decrease.

\section{REFERENCES}

Becker, R. A., Chambers, J. M. \& Wilks, A. R. (1988). The NEW S Language. Pacific Grove, California: Wadsworth \& Brooks-Cole.
Box, G. E. P. \& Jenkins, G. M. (1970). Time Series Analysis: Forecasting and Control. Oakland, California: HoldenDay. 
Cullis, B. R. \& Gleeson, A. C. (1989). Efficiency of neighbour analysis for replicated variety trials in Australia. Journal of Agricultural Science, Cambridge 113, 233-239.

Cullis, B. R. \& Gleeson, A. C. (1991). Spatial analysis of field experiments - an extension to two dimensions. Biometrics 47, 1449-1460.

Cullis, B. R., Gleeson, A. C., Lill, W. J., Fisher, J. A. \& READ, B. J. (1989). A new procedure for the analysis of early generation variety trials. Applied Statistics 38, 361-375.

Cullis, B. R., McGilchrist, C. A. \& Gleeson, A. C. (1991) Error model diagnostics in the general linear model relevant to the analysis of repeated measures and field experiments. Journal of the Royal Statistical Society, Series B 53, 409-416.

FALCONER, D. S. (1981). Introduction to Quantitative Genetics, 2nd edition. New York: Longman.

Gleeson, A. C. \& Cullis, B. R. (1987). Residual maximum likelihood (REML) estimation of a neighbour model for field experiments. Biometrics 43, 277-288.

Kempton, R. A. (1984). The design and analysis of unreplicated field trials. Vorträge für Pflanzenzüchtung 7, 219-242.

Kempton, R. A. \& Howes, C. W. (1981). The use of neighbouring plot values in the analysis of variety trials. Applied Statistics 30, 59-70.
Lin, C. S. \& Poushinsky, G. (1985). A modified augmented design (type 2) for rectangular plots. Canadian Journal of Plant Science 65, 743-749.

MARTIN, R. J. (1985). Some theoretical results on the design and analysis of experiments with second-order stationary spatially correlated errors. Proceedings of the Biometric Society Workshop on Spatial Methods for Field Experiments (Ed. R. A. Kempton), pp. 22-23. Durham: University of Durham.

Patterson, H. D. \& Hunter, E. A. (1983). The efficiency of incomplete block designs in National List and Recommended cereal variety trials. Journal of Agricultural Science, Cambridge 101, 427-433.

TukEY, J. W. (1977). Exploratory Data Analysis. Reading, Mass. : Addison-Wesley.

RICHEY, F. D. (1926). The moving mean as a basis for measuring correlated variation in agronomic experiments. Journal of Agricultural Research 32, 1161-1175.

Williams, E. R. \& LucketT, D. J. (1988). The use of uniformity data in the design and analysis of cotton and barley variety trials. Australian Journal of Agricultural Research 39, 339-350.

YATES, F. (1940). Modern experimental design and its function in plant selection. Empire Journal of Experimental Agriculture 8, 223-230. 